\section{MS6-O5 Covalent host-targeting by thioester domains of Gram-positive pathogens}

Miriam Walden ${ }^{1}$, John M. Edwards ${ }^{2}$, Aleksandra M. Dziewulska ${ }^{2}$, Rene Bergmann ${ }^{3}$, Gerhard Saalbach ${ }^{1}$, Manfred Rohde ${ }^{3}$, Uli Schwarz-Linek ${ }^{2}$, Mark J. Banfield

1. John Innes Centre, Norwich, UK

2. University of St Andrews, UK

3. Helmholtz Centre for Infection Research, Germany

email: miriam.walden@jic.ac.uk

Gram-positive pathogens are a major health concern, with a lack of preventative therapeutics and the ever-increasing resistance to antimicrobials. One of the most crucial steps in infection is adhesion of the microbes to host tissues. Understanding the specific interactions at the host-microbe interface is vital in the development of novel strategies to combat disease. The discovery of complex cell-surface associated proteins, such as pili, has advanced our knowledge of this interaction, however the precise molecular mechanisms underlying the adhesion process remain unclear.

To date, these adhesins are only known to bind host cell receptors in a non-covalent manner. However, recent studies of a Streptococcus pyogenes pilus adhesin revealed the presence of an extremely rare internal thioester bond between the side chains of a Cys and a Gln residue. Mutation of this Cys to Ala results in a $75 \%$ reduction in adhesion to $\mathrm{HaCaT}$ cells, suggesting that this reactive internal linkage may mediate direct attachment.

We have now discovered that thioester domains (TEDs) are unexpectedly prevalent in cell-surface proteins of several clinically relevant Gram-positive pathogens. Using a combination of mass spectrometry and crystallography, we have confirmed the presence of the thioester bond within a selection of twelve TEDs. Furthermore, we show that for the streptococcal surface protein SfbI, this bond can be used as a 'chemical harpoon' to mediate covalent interaction with the host cell protein, Fibrinogen. This cross-linking reaction allows bacterial attachment to fibrin and SfbI binding to human epithelial cells. These findings support bacterial-encoded covalent binding as a new molecular principle in host-microbe interactions.

Keywords: Adhesion, pili, thioester bond, crystallography
MS7. Nucleic acids and their complexes and assemblies with proteins

Chairs: Miquel Coll, Christine Cardin

MS7-01 Translational regulation of gene expression by Lin 28 and Roquin

Udo Heinemann ${ }^{1}$, Yasuhiro Murakawa ${ }^{1}$, Markus Landthaler ${ }^{1}$, Florian Mayr ${ }^{1}$, Anja Schütz ${ }^{1}$

1. Max-Delbrück Center for Molecular Medicine, Robert-Rössle-Str. 10, 13125 Berlin (Germany)

email: heinemann@mdc-berlin.de

RNA-binding proteins contribute to gene expression control by regulating the biogenesis of microRNAs and mRNA homeostasis. Recently, we have studied structural aspects of RNA binding of two important human RNA-binding proteins, Lin28 and Roquin.

Lin28 regulates the maturation of let -7 microRNAs and binds to a large number of mRNAs (1). A Lin28-let-7 regulatory axis is involved in mediating cell differentiation or pluripotency, and LIN28 is overexpressed in a number of cancers. We have analyzed the binding of Lin28 to pre-let-7 molecules by X-ray crystallography and biochemical approaches and provided evidence for an RNA binding model where the Lin 28 cold-shock domain remodels the pre-let-7 structure in order to allow subsequent and sequence-specific binding of the Lin28 zinc-knuckle domain (2). Combined with other studies of the Lin28-let-7 interaction $(3,4)$ this work provides a structural framework for the function of Lin28 in translation-level gene regulation.

Roquin proteins recognize a conserved class of stem-loop RNA degradation motifs, leading to mRNA deadenylation. We have determined the crystal structure of the ROQ domain of human Roquin1/RC3H1 and revealed a mostly helical fold bearing a winged helix-turn-helix (wHTH) motif (5). Through biochemical and mutational analyses we demonstrate that the wHTH motif is involved in binding stem-loop mRNAs that carry constitutive decay elements. Being part of a recent deluge of Roquin structural studies (5-8) our work contributes to putting the biological function of Roquin proteins on a solid mechanistic basis.

\section{References}

1. Mayr, F., Schütz, A., Döge, N. \& Heinemann, U. (2012) Nucleic Acids Res. 40, 7492-7506.

2. Graf, R. et al. (2013) RNA Biol. 10, 1146-1159.

3. Nam, Y., Chen, C., Gregory, R.I., Chou, J.J. \& Kim, V.N. (2011) Cell 147, 1080-1091.

4. Mayr, F. \& Heinemann, U. (2013) Int. J. Mol. Sci. 14, 16532-16553. 
5. Schuetz, A., Murakawa, Y., Rosenbaum, E., Landthaler, M. \& Heinemann, U. (2014) Nat. Commun. 5:5701.

6. Schlundt, A. et al. (2014) Nat. Struct. Mol. Biol. 21, 671-678.

7. Tan, D., Zhou, M., Kiledjian, M. \& Tong, L. (2014) RNA. Nat. Struct. Mol. Biol. 21, 679-685.

8. Srivastava, M. et al. (2015) Nat. Commun. 6:6253.

Keywords: Lin28. Roquin, RNA binding
MS7-O2 Recombinant multiprotein complex production: identification of a novel

building block of the general transcription factor TFIID

Simon Trowitzsch ${ }^{1}$

1. Institute of Biochemistry, Goethe Universität Frankfurt

email: trowitzsch@biochem.uni-frankfurt.de

Multiprotein complexes are a cornerstone of biological activity, as many proteins appear to participate stably or transiently in large multisubunit assemblies. Analysis of the architecture of these assemblies and their manifold interactions is imperative for understanding their function at the molecular level. Powerful recombinant protein production technologies, e.g. MultiBac - a baculoviral expression vector system for multiprotein complex production, are constantly being developed to produce material in great enough quantities for structural and functional studies.

Here, I will present our recent work ${ }^{1}$ on the general transcription factor IID (TFIID), which plays a key role in RNA polymerase II transcription initiation in eukaryotic cells. Human TFIID is a megadalton-sized multiprotein complex composed of the TATA-binding protein (TBP) and 13 TBP-associated factors (TAFs). How these individual proteins assemble into a functional transcription factor is poorly understood to date. We identified a heterotrimeric TFIID subcomplex consisting of the TAF2, TAF8 and TAF10 proteins, which assemble in the cytoplasm. This heterotrimeric TAF complex was produced recombinantly in insect cells using the MultiBac system. By means of native mass spectrometry, we defined the interactions between the TAFs and uncovered a central role for TAF8 in nucleating the complex. X-ray crystallography reveals a non-canonical arrangement of the TAF8-TAF10 histone fold domains. Binding assays including peptide arrays and surface plasmon resonance experiments showed that TAF2 binds to multiple motifs within the TAF8 C-terminal region. These interactions direct the incorporation of TAF2 into a core-TFIID complex that exists in the nucleus. Our results provide evidence for a stepwise assembly pathway of nuclear holo-TFIID, regulated by nuclear import of preformed cytoplasmic submodules.

1) Trowitzsch S, Viola $C$, Scheer E, Conic S, Chavant V, Fournier M, Papai G, Ebong IO, Schaffitzel C, Zou J, Haffke M, Rappsilber J, Robinson CV, Schultz P, Tora L, and Berger I. (2015) Cytoplasmic TAF2-TAF8-TAF10 complex provides evidence for nuclear holo-TFIID assembly from preformed submodules. Nature Communications. 6:6011.

Keywords: multiprotein complexes, transcription, TFIID, BEVS, MultiBac 